\title{
Anisotropic permittivity of ultra-thin crystalline Au films: Impacts on the plasmonic response of metasurfaces
}

\author{
Sawyer D. Campbell, ${ }^{1, a)}$ Richard W. Ziolkowski, ${ }^{1,2}$ Jiangrong Cao, ${ }^{3}$ Slimane Laref, ${ }^{4}$ \\ Krishna Muralidharan, ${ }^{4}$ and Pierre Deymier ${ }^{4}$ \\ ${ }^{1}$ College of Optical Sciences, University of Arizona, 1630 E. University Blvd., Tucson, Arizona 85721, USA \\ ${ }^{2}$ Department of Electrical and Computer Engineering, University of Arizona, 1230 E. Speedway Blvd., Tucson, \\ Arizona 85721, USA \\ ${ }^{3}$ Optics Research Laboratory, Canon U.S.A. Inc., 9030 S. Rita Road, Tucson, Arizona 85747, USA \\ ${ }^{4}$ Department of Material Science and Engineering, University of Arizona, 1235 E. James E. Roger Way, \\ Tucson, Arizona 85721, USA
}

(Received 21 June 2013; accepted 16 August 2013; published online 27 August 2013)

\begin{abstract}
It has been determined by density functional theory (DFT) simulations that the extracted permittivities of ultra-thin crystalline gold $\mathrm{Au}$ ) films exhibit large anisotropies which are not predicted by classical models or previous experimental determinations of the dielectric function. The optical scattering characteristics of a periodic array of Au discs are simulated with the DFT extracted permittivity and contrasted against those obtained with several commonly used Au permittivity models. It is demonstrated that the DFT-based transmittance spectra for these plasmonic metasurfaces lead to significantly redshifted results when compared to those predicted by standard Drude and Johnson-Christy permittivity models. (C) 2013 AIP Publishing LLC.

[http://dx.doi.org/10.1063/1.4819770]
\end{abstract}

Recent advancements in metamaterial research have opened the possibility for new and exciting applications which are not possible with conventional materials. As new advancements have been made, the desire to design metamaterials which operate at optical frequencies has increased dramatically. Due to improvements in nano-scale manufacturing capabilities, there has been a surge of optical metamaterial $^{1}$ and metasurface (frequency selective surface (FSS)) designs. ${ }^{2}$ As nano-scale manufacturing continues to improve and optical devices continue to shrink in size, our understanding of material properties at the nanoscale must improve.

In this study, we present plasmonic enabled frequency selective surfaces, i.e., optical filters, based on metasurface (FSS) structures that are constructed from ultra-thin gold (Au) films whose permittivities have been determined through density functional theory (DFT) simulations ${ }^{3}$ and confirmed experimentally. ${ }^{4}$ These ultra-thin Au films possess large anisotropies in their DFT-based permittivities which are not predicted by classical models. We contrast the resonance behaviors of metasurface (FSS) structures using the DFT-based permittivities, ${ }^{3}$ a commonly used Drude model, the experimentally obtained Johnson-Christy (JC) values, ${ }^{5}$ and a size-dependent permittivity (SDP) model based on the JC data which incorporates a size-dependent dampening term and inter-band transitions. ${ }^{6}$

The DFT model explicitly takes into account the sizedependent electronic band-structure of the Au nanostructures from which an effective permittivity of the true nanoscale crystalline Au thin film is extracted. Specifically, the DFT data used in this work was obtained for (111) Au films, using the independent particle approximation (IPA) framework implemented within the Vienna $\mathrm{Ab}$ initio Simulation

${ }^{\text {a)} E l e c t r o n i c ~ m a i l: ~ s c a m p b e l l @ o p t i c s . a r i z o n a . e d u . ~}$
Package (VASP). ${ }^{7}$ Such an approach has already been adopted to verify the optical properties of bare copper substrates. ${ }^{8}$ Here, the interband contribution to the optical permittivity is obtained by evaluating direct electronic transitions between two single particle energies belonging to two different energy bands in the limit of a vanishing photon wave-vector within the first Brillouin zone. Similarly, the intraband contribution is determined by the free-electron plasma frequency, which is related to the strength of the electronic transitions between partially occupied states, contained within a single band, that are below and above the Fermi-level, respectively.

One interesting property of the Au films that has been revealed by from these DFT simulations is the appearance of a strong anisotropy in the ultra-thin Au films which is not present in any of the traditional permittivity models of $\mathrm{Au}$. Fig. 1 compares the behaviors with respect to the excitation wavelength of the real and imaginary components of the permittivity functions of a 5.0894 nm-thick Au film, which corresponds to 22 atomic monolayers with orientation (111), predicted by the DFT model and the corresponding values generated by the Drude, SDP, and JC models. Crystalline plasmonic films this thin are becoming an experimental reality. ${ }^{9}$ As will be demonstrated with simulations of a metasurface (FSS) device, this anisotropic behavior has a significant impact on its performance as an optical filter.

It is clear from Fig. 1 that the longitudinal $(z z)$ component of the DFT permittivity differs greatly from its transverse ( $x x$ and $y y)$ components. This is a direct consequence of the fact that for the longitudinal, out-plane $(z z)$ component, the dominant contributions arise from interband transitions, while the intraband contributions are negligible. This behavior can be attributed to the highly confined dimension of this out-of-plane direction. The difference between the DFT results and the other models is pronounced in both the 


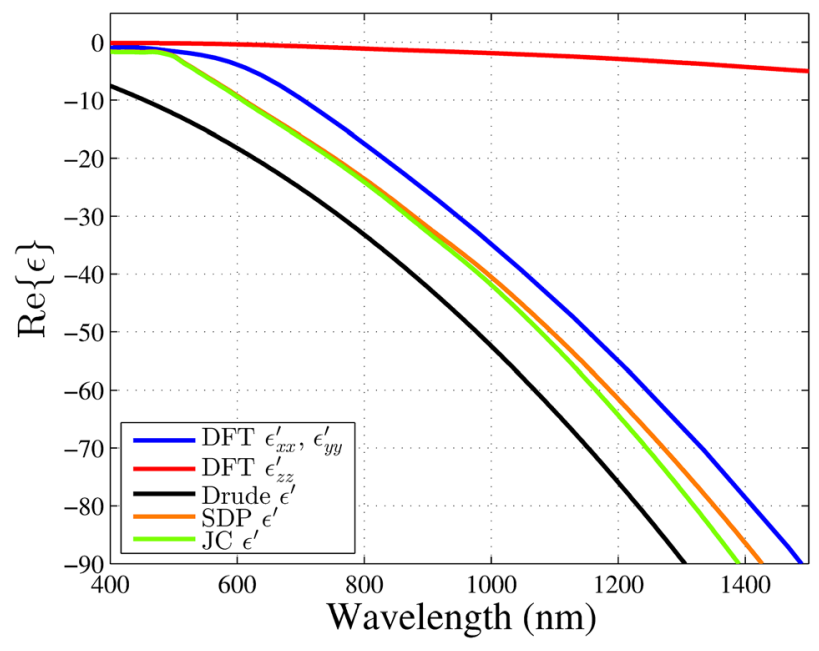

(a) Real part

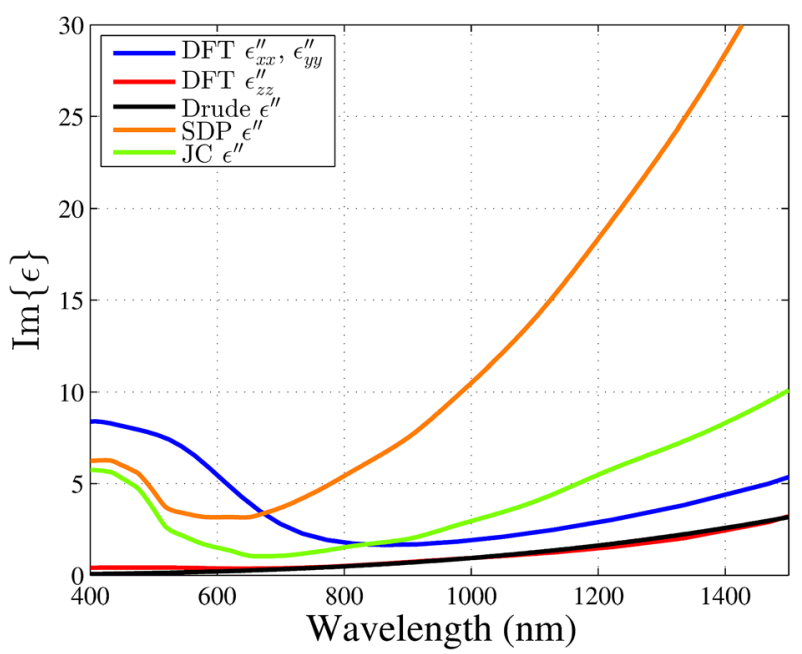

(b) Imaginary part

FIG. 1. Real (a) and imaginary (b) parts of the permittivities for the DFT, Drude, SDP, and JC models of Au.

real and imaginary parts of the dielectric function. There is a clear red-shift in the real part of the permittivity as one progresses from the Drude to the JC, to the SDP, and finally to the fully quantum mechanical DFT results. The differences in the behaviors of the imaginary parts are more dramatic. The DFT longitudinal component agrees reasonably well with the Drude model, while its transverse components agree more favorably with the JC values. The SDP values behave similarly to these results within the visible range. If the film thickness were to be decreased further $(<5.0894 \mathrm{~nm})$, the differences between the DFT and JC permittivities become more exaggerated. On the other hand, the permittivity extracted from the DFT simulations does recover the JC data for films of sufficient thickness (approximately $20 \mathrm{~nm}$ ) even though it predicts these significantly different outcomes for the very thin films.

Consider the square unit cell of an infinite metasurface shown in Fig. 2. It is comprised of a circular Au disc that is $5.0894 \mathrm{~nm}$ (22L film thickness) thick, has a radius $r=20 \mathrm{~nm}$, and is centered on the top of a $20 \mathrm{~nm}$ thick silica substrate whose width $w=50 \mathrm{~nm}$. COMSOL multiphysics $\mathrm{v} 4.3$ is

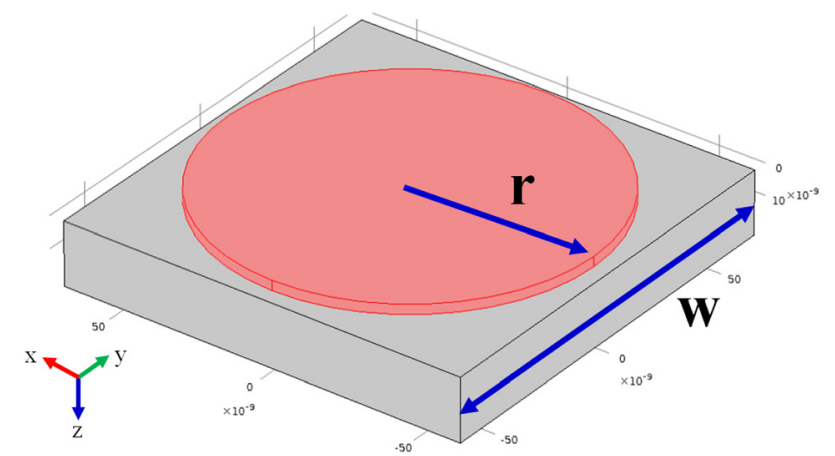

FIG. 2. Isometric view of the square unit cell. The radius of the disc is $r$ and the width of the cell is $w$.

used to model the resonance behavior of this metasurface. The unit cell was excited by an $x$-polarized incident planewave traveling in the $+z$ direction, i.e., by a linearly polarized, normally incident plane-wave. Perfect electric (PEC) and perfect magnetic (PMC) side-walls were used to simulate the infinite planar array. Finally, a full permittivity tensor was implemented to model the anisotropic DFT permittivity. The transmittance results for this metasurface are shown in Fig. 3.

While the Drude-based results show a strong resonance, i.e., no transmittance, in the visible regime at $593 \mathrm{~nm}$, the JC and Drude results are red-shifted and their resonances are not as strong. This red-shifting of the transmittance curves for the JC, SDP, and Drude permittivities follows the trends of the real part of their permittivities noted in Fig. 1(a). On the other hand, the DFT-permittivity shows an even further red-shifting and detuning of the resonance. Even though the performance of the $20 \mathrm{~nm} / 50 \mathrm{~nm}$ metasurface based on the anisotropic DFT permittivity is poor in the visible, moving to larger discs shifts the resonance into the infrared regime where the film has a smaller phase thickness. Consequently, the anisotropic nature has less of an impact on the performance and the losses of the $x x$ and $y y$ components are significantly decreased.

To illustrate this infrared behavior, we considered an enlarged version of the unit cell shown in Fig. 2. It is



FIG. 3. Transmittance for the $20 / 50 \mathrm{~nm}$ metasurface based on the Drude (green), SDP (blue), JC (cyan), and DFT (red) permittivities. 


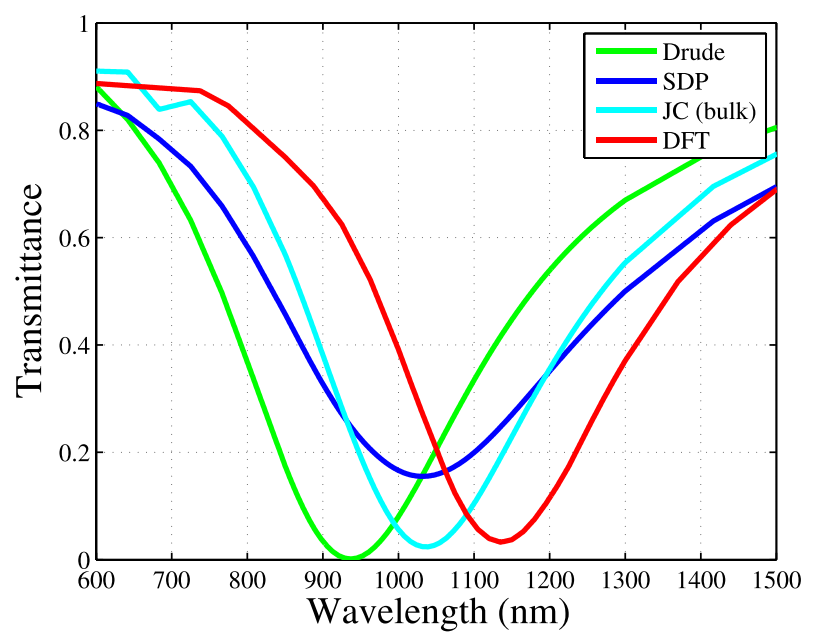

FIG. 4. Transmittance for the $60 / 140 \mathrm{~nm}$ metasurface based on the Drude (green), SDP (blue), JC (cyan), and DFT (red) permittivities.

comprised of a Au disc, whose thickness is $5.0894 \mathrm{~nm}$ and whose radius $r=60 \mathrm{~nm}$, that is centered over a square, $20 \mathrm{~nm}$ thick silica substrate whose width $w=140 \mathrm{~nm}$. This structure was chosen because it provided a compromise between operating at the shortest wavelength possible while maintaining a nearly complete gap in the transmittance response for the DFT-based permittivity. Results for this unit cell based on the Drude, SDP, JC, and DFT permittivities are contrasted in Fig. 4.

Again there is a clear red-shifting of the resonance from the Drude to the JC to the DFT permittivities. The SDP permittivity results correspond more naturally to the JC results; the depth of its transmittance null is noticeably reduced due to the overestimation of the losses in the infrared regime when compared to the JC and DFT permittivities in Fig. 1(b). Again, the ubiquitous Drude and JC permittivities incorrectly predict the behavior of these ultra-thin Au films.

While the anisotropic nature of the DFT permittivity does affect the transmittance behavior of DFT-based metasurfaces, the resonance behavior is surprisingly robust even at large $\left(\sim 45^{\circ}\right)$ angles of incidence. The transmittance behavior of the $60 / 140 \mathrm{~nm}$ DFT-based metasurface for oblique incidence is summarized in Fig. 5. Naturally, the s-polarization case is less sensitive to the anisotropy because the electric field is aligned with the planar ( $x x$ and $y y$ ) components of the permittivity. Even though the gold is very thin, the p-polarization case increasingly "sees" more of the longitudinal $(z z)$ component of the permittivity (and, consequently, the transmittance values become radically different from films whose permittivity is described by an isotropic model based only on the tangential components) at progressively larger oblique angles. Thus, the resonance behavior degrades more quickly for the p-polarization conditions than it does for the s-polarization case.

The electric field distributions and powerflow behavior of the 60/140 nm metasurface are compared in Fig. 6 for the Drude and DFT permittivities. In both cases, the fields are highly localized at the edges of the discs due to the dipole resonance that occurs. The narrow spacing of the discs actually improves the localization of the fields and thus, the magnitude of the transmittance dip. While both the Drude and DFT permittivities achieve a similarly strong dip in the transmittance at resonance, as shown in Fig. 4, the powerflow behavior is quite distinct between these two cases. These differences arise because of the DFT-predicted anisotropy. The streamlines of the Poynting vector field penetrate through the disc and wrap strongly around the poles for the Drude case. On the other hand, the streamlines dissipate as they approach the poles for the DFT case. This is a direct consequence of the anisotropic permittivity and the increased absorption, i.e., the $30.20 \%$ versus $7.04 \%$ absorption for the DFT and Drude cases, respectively.

The accuracy of predicting the behavior of any metamaterial or metamaterial-based device is extremely dependent on the electrical properties of its constituent materials. As nanoscale manufacturing continues to improve, it is important to understand that material properties can vary greatly at the nanoscale in comparison to their bulk counterparts commonly used for much larger dimensions. To emphasize this nano-scale attribute, we have presented here the transmittance behavior of a metasurface device based on the permittivity extracted from DFT simulations of an ultra-thin $\mathrm{Au}$ film and have shown how it significantly differs from that predicted by the standard Drude, JC, and SDP permittivities. Preliminary experiments to validate the anisotropic thin-film effects have been positive. However, we do not have direct experimental data yet which emphasizes the FSS patterning with the single crystal $5.0 \mathrm{~nm}$ thick Au film considered in this paper. We are exploring a potential device level

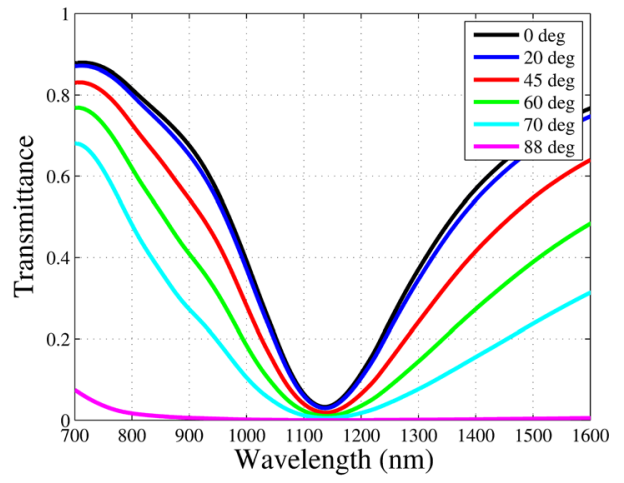

(a) s-polarization

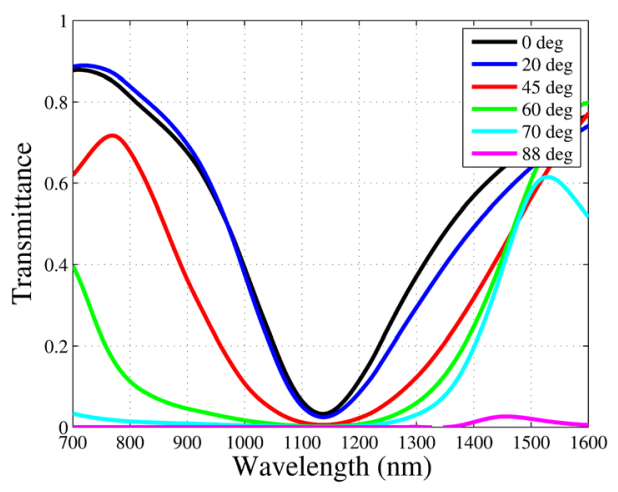

(b) p-polarization
FIG. 5. Transmittance for the $60 / 140 \mathrm{~nm}$ metasurface based on the DFT permittivity for (a) s- and (b) p-polarizations for angles of incidence: $0^{\circ}$ (black), $20^{\circ}$ (blue), $45^{\circ}$ (red), $60^{\circ}$ (green), $70^{\circ}$ (cyan), $88^{\circ}$ (magenta). 


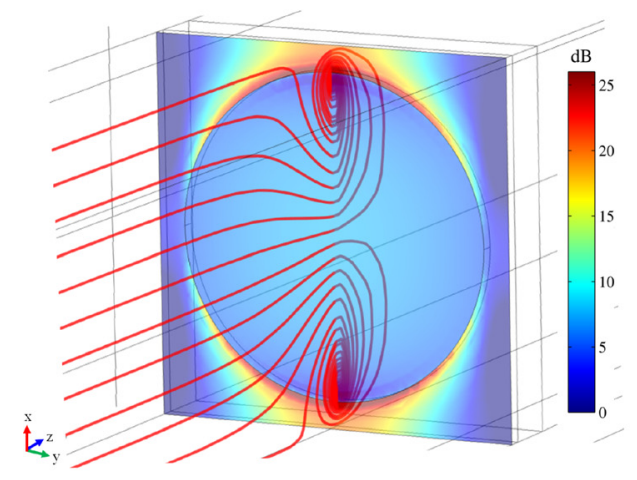

(a) Drude FSS powerflow

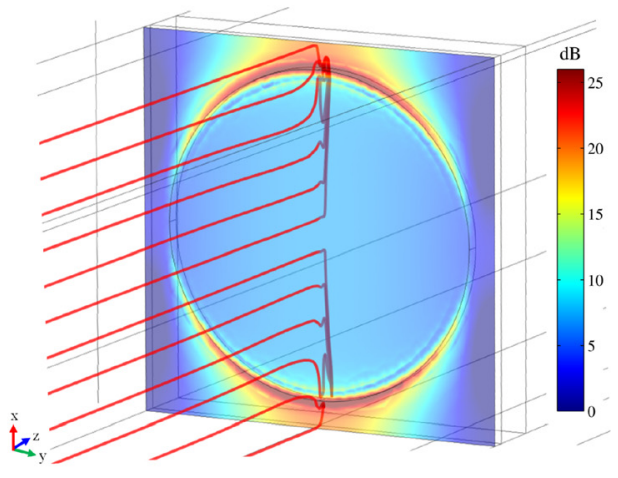

(b) DFT FSS powerflow
FIG. 6. Electric field distributions along with streamlines of the Poynting's vector field for the metasurface at the resonance wavelengths: $943 \mathrm{~nm}$ and $1153 \mathrm{~nm}$, respectively, for the Drude (a) and DFT (b) permittivities. experiment that would measure the oblique incidence transmittance spectra for s- and p-polarizations to validate the results given in Fig. 5 and to confirm the predicted impact of the underlying anisotropic permittivity behavior. We hope to provide a complete synopsis of these results in the near future.

${ }^{1}$ N.-H. Shen, T. Koschny, M. Kafesaki, and C. M. Soukoulis, Phys. Rev. B 85, 075120 (2012).

${ }^{2}$ J. Zhang, J.-Y. Ou, N. Papasimakis, Y. Chen, K. F. MacDonald, and N. I. Zheludev, Opt. Express 19, 23279 (2011).
${ }^{3}$ S. Laref, J. Cao, A. Asaduzzaman, K. Runge, P. Deymier, R. W. Ziolkowski, M. Miyawaki, and K. Muralidharan, Opt. Express 21, 11827 (2013).

${ }^{4}$ J. Cao, R. Balachandran, M. Keswani, K. Muralidharan, S. Laref, R. W. Ziolkowski, K. Runge, P. A. Deymier, S. Raghavan, and M. Miyawaki, Proc. SPIE 8632, 86321I (2013).

${ }^{5}$ P. B. Johnson and R. W. Christy, Phys. Rev. B 6, 4370 (1972).

${ }^{6}$ J. A. Gordon and R. W. Ziolkowski, Opt. Express 15, 2622 (2007).

${ }^{7}$ G. Kresse and J. Furthmller, Comput. Mater. Sci. 6, 15 (1996).

${ }^{8}$ J. Harl, G. Kresse, L. D. Sun, M. Hohage, and P. Zeppenfeld, Phys. Rev. B 76, 035436 (2007)

${ }^{9}$ Y. Lu, J. Kim, H. Chen, C. Wu, N. Dabidian, C. Sanders, C. Wang, M. Lu, B. Li, X. Qiu, W. Chang, L. Chen, G. Shvets, C. Shih, and S. Gwo, Science 337, 450 (2012). 